\title{
Rational Use of Cholinesterase Activity Testing in Pesticide Poisoning
}

\author{
James E. Lessenger, MD, and Benjamin E. Reese, PhD
}

Background: The use of acetylcholinesterase (AChE) activity testing in pesticide poisoning often falls on family physicians when evaluating a suspected poisoning or when monitoring the health of pesticide applicators.

Methods: A review of the literature and consideration of three illustrative cases shows misunderstandings in the pathophysiology of the enzyme and in procedures for effective testing and monitoring of AChE levels.

Results and Conclusions: The physiologic characteristics of acetylcholine neurotransmission are described and related to carbamate and organophosphate poisoning. Pre-exposure monitoring is described using the California plan. A 23 percent variance in AChE levels exists among normal patients. It is necessary, therefore, to establish baseline levels to overcome individual variance. The practice of measuring of $\mathrm{AChE}$ levels in acute poisoning is limited. In employees who have been monitored and for whom baseline AChE levels have been established, a diagnosis of poisoning can be made by comparing postexposure AChE levels with baseline levels. If there is no baseline level recorded, and if the offending chemical is in question, the clinician must base treatment on the clinical signs and symptoms. (J Am Board Fam Pract 1999;12:307-14.)

Thousands of tons of acetylcholinesterase (AChE)inhibiting carbamate and organophosphate pesticides are used throughout the world for agricultural applications as an insecticide, acaricide, aphicide, larvicide, and nematocide. A few organophosphates are used as herbicides. In addition, four organophosphates have military applications as means of mass destruction, and sarin has recently been used as a terrorist weapon. ${ }^{1,2}$

Direct measurement of pesticide levels in blood or urine is cumbersome and expensive. Each pesticide requires a separate assay, and the serum level of the chemical might not be directly related to the degree of enzyme poisoning. Furthermore, even in witnessed exposures that produce symptoms and signs, blood chemical levels can still be too low for detection. Cholinesterase activity testing, however, has the advantage of delivering a measure of physiologic response. ${ }^{3,4}$

In this article we review cholinesterase testing after carbamate and organophosphate exposure from the standpoint of physiology, laboratory testing, pre-exposure monitoring, and diagnosis.

Submitted, revised, 30 July 1998.

From a private practice (JEL), and the Neuroscience Research Institute and Department of Psychology (BER), University of California, Santa Barbara. Address reprint requests to James E. Lessenger, MD, 841 West Morton St, Porterville, CA 93257.

\section{Physiology}

Acetylcholine transmits electrochemical impulses across neuronal synapses and neuromuscular junctions and is hydrolyzed by the action of the enzyme acetylcholinesterase (AChE). The toxic effects of carbamate and organophosphate agents result from their ability to inhibit the catalytic activity of $\mathrm{AChE}$ in the nervous system by forming covalent bonds to acetylcholine receptors and thus preventing hydrolysis of acetylcholine by the enzyme.

The complexes that are formed between these poisons and the enzyme are hydrolyzed slowly in the case of the carbamoylated enzymes or not at all with some phosphorylated enzymes, thereby prolonging the action of acetylcholine. Enzyme activity returns only after a period of days or weeks, when new AChE molecules are synthesized. ${ }^{5}$

Genetic influences not related to sex, race, or age account for a 23 percent variation in $\mathrm{AChE}$ activity levels among humans. Two types of AChE receptors exist: (1) nicotinic, which are always excitatory; and (2) muscarinic, which produce either an excitatory or inhibitory postsynaptic potential. ${ }^{6}$

Acetylcholine is used by all preganglionic neurons of the autonomic nervous system and by the ganglionic cells of the parasympathetic division, innervating pupillary muscles, lacrimal 
Table 1. Signs and Symptoms of AcetylcholinesteraseInhibiting Agent Poisoning.

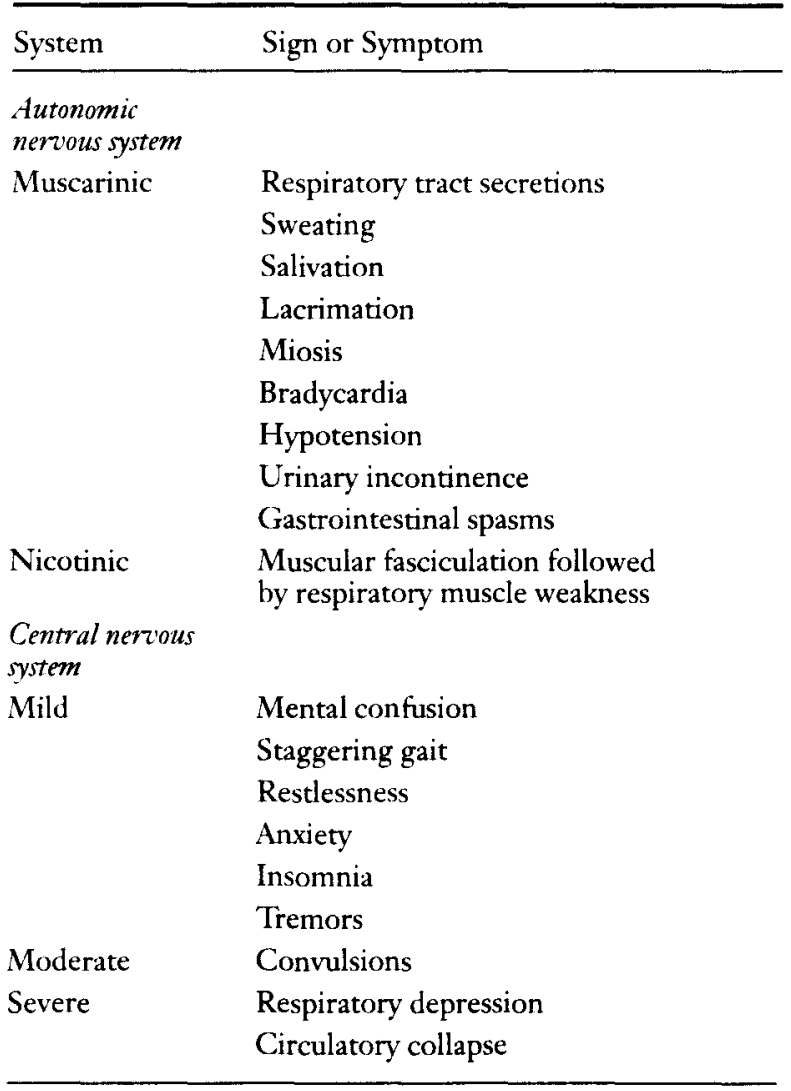

glands, salivary and mucosal glands of the oral and nasal cavities, and cardiac and smooth muscle of various organs. ${ }^{7}$

Cholinergic transmission in all autonomic ganglia is mediated by nicotinic receptors. Postganglionic action, by contrast, is mediated by muscarinic receptors found in smooth and cardiac muscle and in the exocrine glands. ${ }^{7}$

Prolonged nicotinic receptor activation in striated muscle leads to muscle twitching and weakness and to a variety of muscarinic symptoms including sweating, salivation, tearing, blurred vision, pinpoint pupils, decreased heart rate and blood pressure, bronchial constriction, and respiratory distress (Table 1$)^{?}$

The effects on the central nervous system of prolonged acetylcholine action by organophosphates and carbamates include restlessness, tremors, staggering gait, mental confusion seizures, cardiovascular failure, and respiratory depression (Table 1 ). ${ }^{7}$ Among long-term pesticide applicators, there is evidence of reduced cognitive function even when clinical symptoms of excessive cholinergic activity are not present.

\section{Laboratory Testing}

Six methods of determining AChE activity have been developed; of these, the electrometric method, which measures a $\mathrm{pH}$ change, and the colorimetric method are most often used. Both methods are effective for serum and erythrocyte testing and are relatively simple, inexpensive, and reproducible. $^{8}$

Even with modern testing kits and methods, the determination of serum and erythrocyte $\mathrm{AChE}$ activity levels is highly dependent upon technician experience and skill. There are two reporting methods giving two- and five-digit responses, and although there are conversion methods between the two reporting systems, these methods are subject to error. ${ }^{9}$

Aware of such sources of error, the State of California, the only jurisdiction requiring $\mathrm{AChE}$ testing for pesticide applicators, requires laboratory certification. ${ }^{10}$ The following case is illustrative of laboratory problems encountered in cholinesterase testing.

\section{Case 1}

A 32-year-old man referred for consultation worked as a irrigator for a large agribusiness firm. When he was promoted to a better paying job as a pesticide applicator, $\mathrm{AChE}$ testing was conducted to establish a baseline measurement.

The supervising physician sent all blood samples to laboratory $M$. All AChE tests were reported by the laboratory as abnormal with results of $1119 \mathrm{U} / \mathrm{L}, 1884 \mathrm{U} / \mathrm{L}$, and $1036 \mathrm{U} / \mathrm{L}$ for serum (normal 2900 to $7100 \mathrm{U} / \mathrm{L}$ ), and $37 \mathrm{U} / \mathrm{g}, 35 \mathrm{U} / \mathrm{g}$, and $37 \mathrm{U} / \mathrm{g}$ of hemoglobin for erythrocytes (normal 24 to $40 \mathrm{U} / \mathrm{g}$ ). Because the serum levels were less than normal, the company sent blood samples to a second laboratory, which reported levels of $985 \mathrm{U} / \mathrm{L}$ for serum (normal 1420 to $5004 \mathrm{U} / \mathrm{L}$ ) and $6242 \mathrm{U} / \mathrm{g}$ for erythrocyte (normal 2900 to $7100 \mathrm{U} / \mathrm{g}$ ) AChE activity. The second laboratory sent results directly to the company; no physician's opinion was given because the laboratory claimed it could act as the supervising physician. When contacted by the company, the local poison control center stated the person must have been exposed to organophosphates and poisoned.

When the employee was examined by a physician, he had no complaints, current or in the past. His pulse rate, respirations, temperature, and blood pressure, as well as results of a complete 
physical examination, were normal. The employee could recall no time when he was exposed to pesticides, and spraying reports showed that he had entered fields sprayed only with herbicides.

Inquiries by telephone found that laboratory $M$ was not state certified, even though it had represented itself as being certified to both the company and the physician. Further, the laboratory used the $\mathrm{pH}$ method for the first test and switched to the colorimetric system for the latter two. Although the second laboratory was state certified, it had not followed the state protocol for the testing, had used more than 1 technician, and was surprised when told it could not function as a supervising physician.

Less than 1 week after the last AChE level was measured, the tests were repeated in a state-certified laboratory using the state protocol and experienced technicians. The first two serum and erythrocyte $\mathrm{AChE}$ levels were in the normal range, but because the first two levels were not within 15 percent of each other, a third level was measured, and the two closest levels were averaged to establish a baseline measurement. That baseline measurement was far higher than those reported by the other laboratories. As will be detailed below, the elapsed time between tests was too short for a depressed $\mathrm{AChE}$ level to have returned to baseline. The original laboratory report was considered to be in error, and the employee returned to full duty.

\section{Case Discussion}

This employee lost 4 weeks of work because the laboratories used the wrong methods and protocols, and a diagnosis was made solely on laboratory test results without taking clinical findings into consideration.

\section{Pre-Exposure Testing}

When evaluating AChE tests clinically, three factors must be kept in mind: (1) anticholinesterase agents depress $\mathrm{AChE}$ levels, (2) baseline levels vary an average of 23 percent among individuals, and (3) results can be affected by extraneous health problems.

It is common for consultants and investigators to encounter patients who have a diagnosis of pesticide injury and have been removed from work for considerable lengths of time as a result of elevated AChE levels. ${ }^{9}$

The normal variation in AChE levels is not re- lated to sex, age, socioeconomic levels, or ethnicity and is unpredictable but measurable. Any monitoring method that does not take this variation into consideration is therefore invalid. If $\mathrm{AChE}$ levels are determined only after an alleged exposure, the possibility of a low but normal AChE activity level could lead to a false-positive finding. ${ }^{6}$

Diseases, medications, and illegal drugs can affect AChE levels. Hepatorenal and neuromuscular disease, as well as wasting from any chronic disease, can cause alterations in blood levels, and alcoholism, including the resulting hepatorenal and neural damage, can affect AChE metabolism. Medications, especially those affecting the neuromuscular junction, such as physostigmine, can alter AChE blood levels. Cocaine can lower AChE levels, which thus contributes to cocaine toxicity and heightens susceptibility to organophosphates and carbamates. ${ }^{11-15}$

The State of California, the leader in $\mathrm{AChE}$ monitoring, has adopted a standard protocol to monitor pesticide applicators, mixer-loaders, flagmen, maintenance personnel, and others in dayto-day contact with carbamates and organophosphates. Participants in the program include employers, who must assure that testing is done; employees, who are tested; physicians, who evaluate the results and make recommendations; statecertified laboratories, which perform the tests; the county agricultural commissioners' office, which oversees employer compliance and investigates accidents; and the Department of Health Services, which monitors program quality (Table 2). ${ }^{10,16}$

Survey results have shown that most monitoring is done incorrectly. Ames and associates ${ }^{16}$ have found four categories of problems with $\mathrm{AChE}$ monitoring: (1) employers fail to refer employees for baseline measurements and monitoring, (2) physicians fail to interpret test results properly and to make appropriate recommendations, (3) the insufficient number of county staff personnel are unable to monitor employers adequately, and (4) the state is unable to monitor physicians because training and certification are not required for a physician to be a medical supervisor. Proposed solutions include employer and physician training as well as a certification program for physicians.

Fillmore and Lessenger ${ }^{17}$ found problems related to employees who were removed from work because of low AChE blood levels. Often employees were simply laid off by their employers and 
Table 2. California Cholinesterase Activity Monitoring Program.

Baseline (repeated every 2 years)

1. Established before the worker begins spraying

2. Procedure

a. Two tests are performed not less than 3 days and not greater than 14 days apart

b. If the 2 test results vary by more than $15 \%$, a third test is performed within 3 to 14 days

c. An average of the two closest values becomes the baseline

Periodic testing, if spraying 6 days in a 30-day period

1. Three tests at 30 -day intervals

2. Then at 60-day intervals for the remainder of the 2 -year period

3. More often at the discretion of the medical supervisor a. If values are inconsistent or low

b.If employee has been involved in an exposure

Action

1. Plasma or erythrocyte cholinesterase level falls to $80 \%$ of baseline: special report to employer

2. Plasma level falls to $60 \%$ of baseline or erythrocyte level falls to $70 \%$ of baseline: remove employee from duty

3. Employee must remain off duty until both serum and erythrocyte cholinesterase levels return to $80 \%$ of baseline value

Adapted from the California Environmental Protection Agency guidelines. ${ }^{10}$

told to apply for unemployment compensation. Several had to take a pay reduction and were assigned to a another job task, or they had to quit their jobs, seek employment elsewhere, and establish new low-postexposure baseline levels. Many employers repeated $\mathrm{AChE}$ baseline measurements at the end of the spraying season to establish low normal blood levels that would not be so likely to be reported as toxic if they became further decreased. There were also seasonal effects upon $\mathrm{AChE}$ levels, with levels lowest in the autumn regardless of spraying history. Furthermore, many clinically ill persons had a 60 percent reduction in their blood levels even though they stayed in the reported normal range of results. All the problems and pitfalls notwithstanding, $\mathrm{AChE}$ monitoring has been shown to detect serious reductions in AChE levels in applicators before clinical illness develops, as illustrated in case 2 .

\section{Case 2}

A pesticide-spraying company sent all 13 of its employees for baseline AChE measurements in April and May of 1992. Six weeks later all 13 employees were found to have depressed AChE levels during a routine check. When questioned, they admitted to problems of dizziness and blurred vision.

Although all 13 complained of vertigo and blurred vision, none complained of salivation, lacrimation, urination, or diarrhea-the so-called SLUD syndrome classically associated with AChE poisoning. None of the employees could recall any particular incident or time when excess exposure occurred. Their blood pressure, temperature, respiratory rate, and pulse rate were normal, and results of blood chemistry analysis and physical examinations were normal.

The employer claimed laboratory error and demanded that the physician not notify the agriculture commissioner's office. Furthermore, the physician was threatened with litigation and loss of the account if he reported the exposure. Nevertheless, the commissioner's office was notified in accordance with state law, and re-analysis of all tests confirmed the initial results.

Investigators discovered that in early June ( 3 weeks after the baseline levels were established) the crew sprayed several walnut groves at night and removed their protective equipment. Later that night, they sprayed in a strong wind that blew high concentrations of organophosphates back in their faces. The owner threatened the employees with violence to keep them quiet.

All of the workers' blood AChE levels were monitored for 2 to 3 months. The workers finally admitted to symptoms, which persisted until their erythrocyte AChE levels started to return to baseline. Plots of serum and erythrocyte AChE levels from the 13 patients are displayed in Figure 1.

\section{Case Discussion}

The serum data in Figure 1 illustrate two points. First, normal AChE baseline values vary widely. In addition to the physiologic variability, a person's prebaseline exposure can vary while working for the same or another company. Second, workers' responses also vary widely, both in the ultimate decrease in AChE levels and in the speed with which their blood levels decrease and recover.

The data for erythrocyte levels in Figure 1 illustrate three points. First, erythrocyte baseline levels vary far less than do serum levels. Second, erythrocyte levels do not decline nearly as much as serum levels and consequently are not as sensitive an assay. Third, when levels do fluctuate, they tend to follow serum levels when declining, 
but return to baseline long before serum levels return.

Serum levels roughly followed symptoms in their decline, but erythrocyte levels tended to follow symptoms in their recovery. Note that the normal ranges and units of measurement seen in Figure 1 (shaded area) are different from those in case 1.

\section{Exposure Testing}

AChE testing is beneficial only for carbamate and organophosphate poisoning, and these agents comprise a minority of the compounds used as pesticides. He and associates, ${ }^{18}$ writing in China, found the problem of mistakenly diagnosing illness caused by carbamate and organophosphate pesticide poisoning based on cholinesterase testing when in reality they were seeing low but normal AChE levels. Several patients nearly died as the result of injudicious use of atropine (a cholinergic antagonist) as treatment for poisoning with pyrethroids, which have no affect on AChE levels.

Many sources have noted only a weak correlation between changes in $\mathrm{AChE}$ activity levels and symptoms or signs of exposure, and only a low correlation between the severity of exposure, signs and symptoms of toxicity, and $\mathrm{AChE}$ levels. The rapidity with which $\mathrm{AChE}$ activity can change is clinically more relevant than actual levels. Consequently, AChE levels should be interpreted with care and should never be the only diagnostic criterion. The possibility of mass psychogenic hysteria can account for a wide constellation of complaints in populations believing they might have been exposed. ${ }^{19-21}$

There are two AChE testing methods to document exposure and recovery in carbamate and organophosphate exposure. The first is ongoing testing in persons with pre-exposure monitoring, and the second is testing in persons without monitoring.

\section{Ongoing Monitoring}

A dip in AChE activity levels is expected in a person who is subject to ongoing monitoring and who has been exposed to carbamate and organophosphate pesticides (Figure 1). During normal spraying activities, it is common to find employees who have AChE levels that drop lower than the cutoff point. Many will be asymptomatic, and the only finding will be depressed laboratory values. Because these workers are in danger of poisoning if they return to spraying duty and suffer exposure to the pesticides, they are removed from spraying duty (but not necessarily from work) until their values return to baseline. ${ }^{12,20}$

It is common for professional sprayers being routinely monitored to have a substantial accidental exposure and not report their exposure because they fear losing their jobs. Their exposure might be discovered only after a drop in AChE levels is detected upon testing and the employee is confronted with the findings. ${ }^{17,22}$

\section{Without Monitoring}

In other scenarios the employee might be exposed to a substantial amount of chemical and have immediate signs and symptoms of poisoning. Depression of $\mathrm{AChE}$ values can be variable and might not correspond to the profundity of clinical findings. The decision to treat should be based on clinical, not laboratory, considerations. ${ }^{22}$

In Japan more than 600 patients were treated for sarin poisoning used by terrorists. The decision to treat was based on clinical findings. AChE testing was useful in follow-up of the exposed persons, and it took up to 3 months before levels stabilized at presumably normal levels. ${ }^{2}$

In patients with a documented carbamate or organophosphate poisoning and with depressed or normal AChE levels, Coye and associates ${ }^{20}$ have found that postexposure monitoring which reflects an increase in activity greater than 20 percent is diagnostic for carbamate and organophosphate poisoning and recovery in an exposed person. AChE levels taken at the time of poisoning and repeated after the exposure in persons who have no potential for re-exposure should start to show a rebound increase in AChE levels within several weeks. The following case illustrates the use of cholinesterase testing when no baseline measurement is available.

\section{Case 3}

A 27-year-old man who owned a citrus nursery was spraying orange trees with a class I organophosphate and not using any protective mask, hat, or coveralls when the hose of his spray rig ruptured, and his clothing became soaked with the chemical. He repaired the hose and continued spraying but developed a headache and dizziness. He did not develop salivation, lacrimation, or urination but 

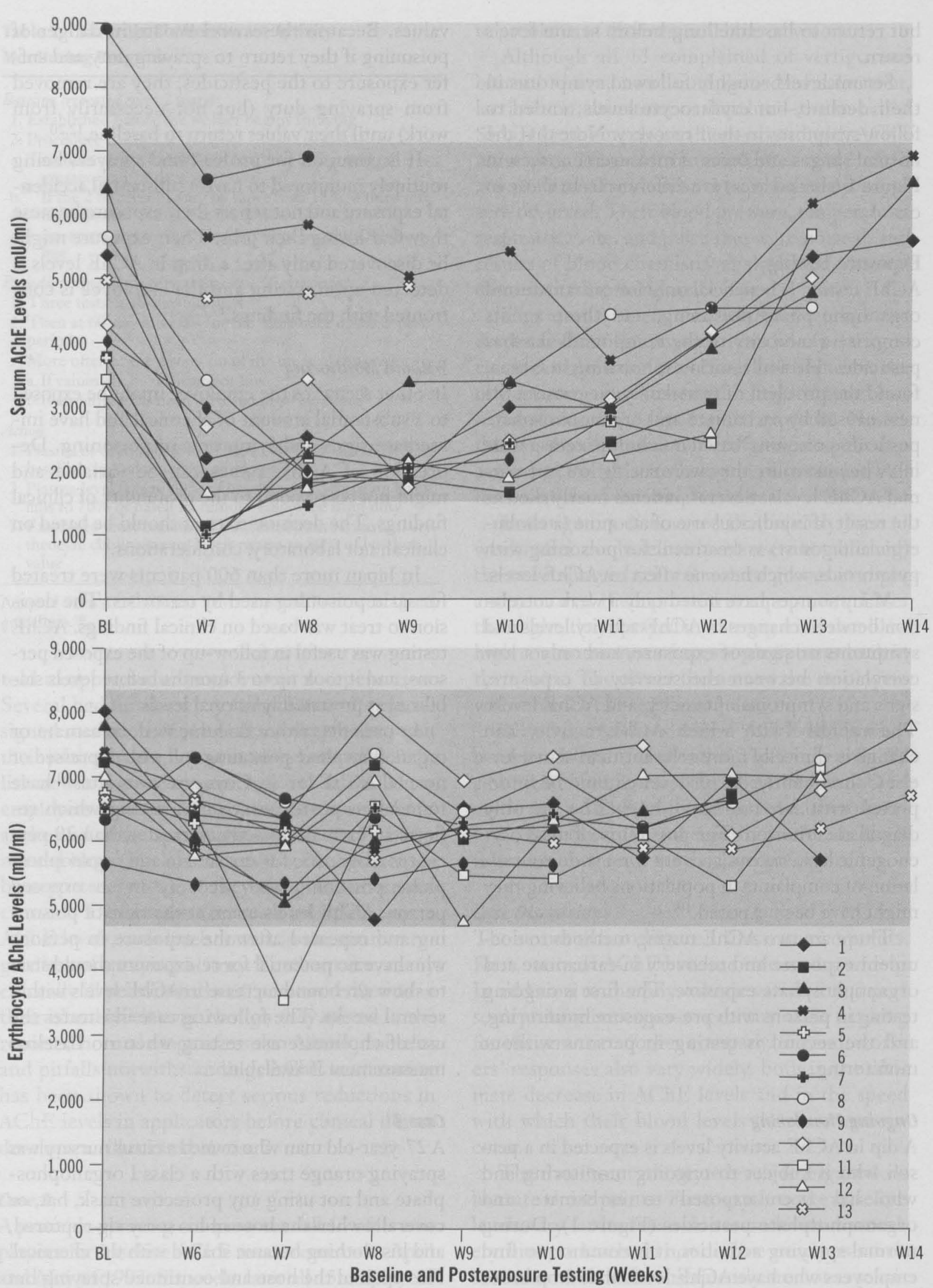

Figure 1. Plots of serum and erythrocyte acetylcholinesterase (AChE) levels from 13 patients. Laboratory normal values are in the shaded area. 
did have loose stools, which he attributed to a recent trip when he consumed contaminated water.

The headaches, dizziness, and anxiety persisted, so he went to an emergency department, where results of an examination were normal and $\mathrm{AChE}$ testing was done. Because the AChE levels were in the normal range, and the patient did not have the classic SLUD syndrome, a diagnosis of anxiety was made and the patient sent to a psychiatrist. No report was made to the agriculture commissioner's office and the farmer continued spraying.

The patient requested a consultation 1 week later. He complained of headaches, dizziness, and loose stools and was extremely anxious about his exposure. Except for a ruddy facial complexion, his temperature, blood pressure, pulse rate, and respiratory rate, as well as results of a physical examination, were normal. Again, his AChE levels were within a normal range, and no comparison could be made with the AChE measurements made in the emergency department because a different method was used.

Subsequent serial AChE activity levels showed a 20 percent increase, although all levels remained within a normal range.

Once the patient was reassured that he was not imagining his illness and that his symptoms were appropriate, his anxiety disappeared. A report was made to the agriculture commissioner's office, and investigators showed him how to use correct safety equipment. After 1 month of using proper application methods, all his symptoms disappeared.

\section{Case Discussion}

The emergency department physician was misled by the patient's normal AChE level even though he had a clear-cut history of exposure and persistent symptoms. The patient did not establish a baseline measurement, as required by law; had he done so, his values probably would have been higher than normal. Pre-exposure baseline values avoid such speculation. The postexposure 20 percent rise in $\mathrm{AChE}$ levels confirmed the diagnosis.

From a clinical standpoint this man's anxiety was also important. Anxiety can be a symptom of organophosphate poisoning as a result of its effects on the central nervous system, but the anxiety could have also been due to the patient's fear concerning his illness, anger that a correct diagnosis could not be made, and fear of being caught by investigators.

\section{Conclusions}

If performed and interpreted correctly, AChE testing can be an important instrument for preventing or diagnosing carbamate or organophosphate poisoning. By understanding the physiologic effects of cholinergic neurotransmission, we know that there is at least a 23 percent variation in $\mathrm{AChE}$ levels among individual patients, that it takes weeks to months for $\mathrm{AChE}$ to regenerate after exposure, and that $\mathrm{AChE}$ levels can be affected by a number of factors other than pesticide exposure.

Pre-exposure monitoring of pesticide applicators has been found to be of benefit in preventing morbidity. Nevertheless, it is important to use a laboratory experienced in AChE testing and to interpret the results by using a standard protocol.

When evaluating possible pesticide poisoning, alternate diagnoses must be kept in mind. The offending pesticide should be identified, if possible; and AChE testing, if performed correctly, can help verify that the chemical is a carbamate or organophosphate.

In the evaluation of a person who has been exposed to an unknown chemical, AChE levels should be interpreted with caution, and long-term monitoring for a rebound effect might be necessary. Treatment should be based on the clinical presentation, not on $\mathrm{AChE}$ levels when a pre-exposure baseline measurement has not been established.

\section{References}

1. Morgan DP. Recognition and management of pesticide poisonings. 4th ed. Washington DC: Environmental Protection Agency, 1989.

2. Morita $H$, Yanagisawa N, Nakajima T, Shimizu $M$. Sarin poisoning in Matsumoto, Japan. Lancet 1995; 346:290-3.

3. Namba T. Cholinesterase inhibition by organophosphorus compounds and its clinical effects. Bull World Health Organ 1971;44:289-307.

4. Ames RG, Brown SK, Mengle DC, Kahn E, Stratton JW, Jackson RJ. Protecting agricultural applicators from over-exposure to cholinesterase-inhibiting pesticides: perspectives from the California programme. J Soc Occup Med 1989;39:85-92.

5. Walker $\mathrm{CH}$. Interactions between pesticides and esterases in humans. In Mackness $\mathrm{MI}$, Clerc $M$, editors. Esterases, lipases, and phospholipases: from structure to clinical significance. New York: Plenum, 1994.

6. Mason $\mathrm{HJ}$, Lewis PJ. Intra-individual variation in plasma and erythrocyte cholinesterase activities and monitoring of uptake of organo-phosphate pesti- 
cides. J Soc Occup Med 1989;39:121-4.

7. Feldman RS, Meyer JS, Quenzer LF. Principles of neuropsychopharmacology. Sunderland, Mass: Sinauer, 1997.

8. Vandekar M. Minimizing occupational exposure to pesticides: cholinesterase determination and organophosphorus poisoning. Residue Rev 1980;75:67-80.

9. Lessenger JE. The California pesticide program: comments from the front lines. J Agromedicine 1996;3:57-68.

10. Guidelines for physicians who supervise workers exposed to cholinesterase-inhibiting pesticides, $3 \mathrm{rd} \mathrm{ed}$. Sacramento: Office of Environmental Health Hazard Assessment, California Environmental Protection Agency, 1995.

11. Lessenger JE. Postulated interaction between hydroxychloroquine and cholinesterase enzyme activity: a case report. J Agromedicine 1995;2:5-12.

12. Hoffman RS, Henry GC, Howland MA, Weisman RS, Weil L, Goldfrank LR. Association between life-threatening cocaine toxicity and plasma cholinesterase activity. Ann Emerg Med 1992;21: 247-53.

13. Simon NM, Del Greco F, Dietz AA, Rubinstein HM. Serum cholinesterase deficiency in renal failure. Trans Am Soc Artif Intern Organs 1969;15:32832.

14. Kutty KM, Jacob JC. Serum cholinesterase activity in hyperlipidemia and the in vitro effect of isoniazid on serum cholinesterase. Can J Biochem 1972:50; $32-4$.

15. Haboudi NA, Thurnham DI. Effect of ethanol on erythrocyte actetylcholinesterase activity. Am Clin Biochem 1986;23(Pt 4):458-62.

16. Ames RG, Brown SK, Mengle DC, Kahn E, Stratton JW, Jackson RJ. Cholinesterase activity depression among California agricultural pesticide applicators. Am J Ind Med 1989;15:143-50.

17. Fillmore CM, Lessenger JE. A cholinesterase testing program for pesticide applicators. J Occup Med 1993;35:61-70.

18. He F, Wang S, Liu L, Chen S, Zhang Z, Sun J. Clinical manifestations and diagnosis of acute pyrethroid poisoning. Arch Toxicol 1989;63:54-8.

19. Lessenger JE, Estock MD, Younglove T. An analysis of 190 cases of suspected pesticide illness. J Am Board Fam Pract 1995;8:278-82.

20. Coye MJ, Barnett PG, Midtling JE, Velasco AR, Romero P, Clements CL, Rose TG. Clinical confirmation of organophosphate poisoning by serial cholinesterase analyses. Arch Intern Med 1987;147: 438-42.

21. Kurtz PH, Esser TE. A variant of mass (epidemic) psychogenic illness in the agricultural work setting. J Occup Med 1989;4:331-4.

22. Lessenger JE. The pesticide-exposed worker: an approach to the office evaluation. J Am Board Fam Pract 1993;6:33-41. 